\title{
Ethical and Safety Concerns Regarding the Use of Mental Health-Related Apps in Counseling: Considerations for Counselors
}

\author{
Kathleen M. Palmer ${ }^{1}$ (D) Vanessa Burrows ${ }^{1}$ \\ Received: 27 December 2019 /Revised: 4 August 2020 / Accepted: 13 August 2020 / Published online: 31 August 2020 \\ (C) Springer Nature Switzerland AG 2020
}

\begin{abstract}
Mental health-related smartphone apps (MHapps) have the potential to greatly enhance and enrich the counseling relationship, and dramatically improve the lives of clients. However, a large portion of MHapps have not been empirically researched and found to be effective. An average of 2 million apps are available in the Apple and Android stores, and users average more than 80 apps on their phones. Many of the apps lack disclaimers about the collection of user information, and there is no governing body to oversee and regulate app development and availability. This is particularly problematic with mental health-related smartphone apps, because many developers are not affiliated with mental health professionals, and many apps do not provide emergency information should a mental health emergency occur while using the app. Moreover, users are left to haphazardly make decisions about health-related apps usage without assistance. Counselors who supplement counseling with mental health-related smartphone apps could unknowingly violate their Code of Ethics by integrating apps that may jeopardize their clients' safety. The authors review literature related to mental health-related app efficacy, safety, and ethics and provide a compilation of items to consider that can be used before supplementing counseling with mental health-related apps.
\end{abstract}

Keywords Smartphone apps $\cdot$ Mental health $\cdot$ Counselor $\cdot$ Code of ethics

\section{Introduction}

The emergence of mobile phones in the twenty-first century drastically altered a massive proportion of individuals' lives. According to the Pew Research Center (2019), 96\% of Americans own a mobile phone, an increase of $35 \%$ since 2011. Of those individuals, $81 \%$ own a smartphone mobile device, which has the capability of searching the internet. Additionally, a major capability of smartphone devices is the ability to download and use a variety of applications (apps). As of the third quarter of the year 2019, Android phone users were able to choose from 2.47 million smartphone apps to download, and Apple phone users with 1.8 million smartphone apps available for download (Clement 2019f). In 2018, 194 billion smartphone apps were downloaded worldwide (Clement 2019a).

On average, individuals have more than 80 apps installed on their smartphone, and $92 \%$ of the time on a smartphone

Kathleen M. Palmer

palmerkm@udmercy.edu

1 University of Detroit Mercy, 4001 W. McNichols Road, Detroit, MI 48221-3038, USA device is spent on apps (Ahmad 2019; Khalaf and Kesiraju 2017). Of the smartphone apps available for download, 318,000 are health apps, which are accessible for smartphone users worldwide (Liquid State 2018). As of May 2019, approximately $1.82 \%$ of the available apps in the Apple App Store are within the category of medical apps (Clement 2019b). These apps contain both medical and/or mental health-related content. Various terms are used interchangeably to describe mental health-related smartphone apps (MHapps), including mHealth apps, mental health apps, health and fitness apps, and medical apps. According to Pappas (2020), there were nearly 20,000 MHapps in the app stores. These apps have the potential to significantly impact the mental health care field, as well as become a useful tool in counseling practices. Many MHapps allow users to track their mood, as well as facilitate communication between them and their mental health counselors without seeing them face-toface (Rouse 2018).

In a study conducted by Krebs and Duncan (2015), more than $50 \%$ of the participants were found to have used an MHapp. However, this number has dramatically increased since the onset of COVID-19 in 2020. According to Herzog (2020), there were 4 million first-time downloads of the top 20 mental wellness apps in April 2020 in the USA, which is an 
increase of 3.1 million (29\%) within 3 months (Herzog 2020). Talkspace, which is a popular text- and video-based therapy app, indicated their number of new users doubled between mid-March and May 1, 2020 (Herzog 2020). This radical increase in first-time downloads and users exemplifies the interest and proliferation of MHapps.

However, research indicates very few MHapps available to consumers are empirically tested, if researched at all (Lui et al. 2017; Larsen et al. 2016). According to Schueller, Executive Director of Psyberguide, only 3 to $5 \%$ of MHapps are research based (Clay 2020). Further complicating this matter is that new apps are added to the smartphone application market at a rate that is faster than research can keep up with (Anthes 2016; Rathbone and Prescott 2017; Watts et al. 2013). For example, between 2016 and 2018, an average of 1434 smartphone apps were released every day in the Apple App Store (Clement 2019e) and 6140 smartphone apps in the Google Play Store between 2016 and 2018 (Clement 2019d). However, considering the dramatic increase in firsttime MHapp downloads previously discussed, it is likely the number of apps released daily increased exponentially during the first quarter of 2020.

Furthermore, another factor affecting MHapp research is that apps lose on average $77 \%$ of their daily active users in just 3 days and users only keep an app for 6 days before losing interest and uninstalling it (Vempaty 2019; Watson 2018). Larsen et al. (2016) conducted a search in the Google play and Apple Store for apps related to depression, bipolar, and suicide. These authors found that more than $50 \%$ of the apps had changed in just 4 months, and an app was removed every 2.9 days. It is likely that this volatile nature of smartphone apps development and the users' short-term loss of interest has impeded researchers' ability to conduct and disseminate up-to-date evidence-based research findings regarding the effectiveness of MHapps.

The COVID-19 pandemic of 2020 has demanded licensed mental health counselors examine alternative ways to provide their clients with counseling services. The integration of technology in mental health counseling has become necessary to accommodate client needs, but the rapid changes may present challenges for licensed mental health counselors. Considering the copious number of and prolific use of apps, it is surprising that there is a dearth of research related to their effectiveness, safety regulations, and ethical issues oriented towards licensed mental health counselors. The majority of recent research regarding the effectiveness, safety, and ethical use of MHapps appear aimed towards the psychiatry and psychology disciplines. For example, see Bakker et al. (2016), Bakker and Rickard (2018), Edwards-Stewart et al. (2019), Jones and Moffitt (2016), Leigh and Flatt (2015), Lindhiem et al. (2015), Lui et al. (2017), Marshall et al. (2019a), Marshall et al. (2019b), Marshall et al. (2020), Prentice and Dobson (2014), Rathbone and Prescott (2017), Reamer (2015),
Stratton et al. (2017), Torous and Roberts (2017), and Wang et al. (2018). Considering licensed mental health counselors are also likely to encounter issues regarding the use of MHapps, it is puzzling why this gap in literature exists.

This article highlights some of the issues licensed mental health counselors should be aware of to help ensure client safety, while avoiding potential liability issues associated with ethical violations. Additionally, in lieu of a research-based tool for counselors to independently assess or evaluate MHapps prior to use, the authors provide a non-exhaustive list of items to consider prior to supplementing counseling with MHapps (see Appendix 1).

\section{Literature Review}

\section{Efficacy of MHapps}

Ideally, MHapps are to be used in conjunction with professional counseling and aide with coping and practice skills learned in counseling; however, most apps lack scientific evidence to support their efficacy. As previously indicated, research found that only 3 to $5 \%$ of MHapps is research based (Clay 2020).

Donker et al. (2013) conducted a study to determine the efficacy of MHapps. The researchers found 5464 abstracts that addressed MHapps. Of these abstracts, only eight articles included scientific trials that examined the effects of MHapps using either pre/posttest or experimental control group design. Furthermore, only four out of eight apps studied were supported by mental health professionals.

Lui et al. (2017) state the use of MHapps in a psychotherapy context looks promising for the future of counseling; however, the lack of scientific research and insufficient empirical support is of great concern. These authors reviewed 21 articles to determine the effectiveness and efficacy of MHapps, and the results demonstrate that there is currently insufficient empirical evidence for MHapps. Of the articles reviewed, none of the studies have been replicated by independent research teams, and $42.9 \%$ of the studies did not include a control group (Lui et al. 2017). Without a control group, it is impossible to differentiate between a successful reduction of symptomatology due to the MHapp or due to the passage of time and increased support outside the app. To increase efficacy and effectiveness of MHapps, it may be more useful for independent research teams, rather than app developers, to conduct the research. Lui et al. (2017) stress the importance for further research into MHapps due to the fact that many smartphone apps available to the public are understudied, or not studied at all.

Anthes (2016) states that although some evidence supporting the use of MHapps is becoming apparent, the research has a long way to go. A majority of the research 
published has been conducted by the app's own developers, rather than more reliable independent researchers. The author also states that MHapps have been tested primarily by pilot studies or randomized trials which are small and unreplicated, but the majority of apps have yet to be tested at all.

Grist et al. (2017) found there was insufficient research evidence to support the effectiveness of MHapps, especially for children and adolescents. This is of great concern because adolescents may be more likely to seek smartphone apps for mental health concerns, because they are a discrete and costeffective method to seek help. With very little clinical effectiveness and empirical data to support these smartphone apps, developers may knowingly or unknowingly take advantage of this vulnerable population. This is of particular importance due to the fact that this population of individual's access smartphone apps 9.4 times per day (Clement 2019c) and a $30 \%$ increase in engagement of non-gaming apps was found in 2018 (The state of mobile in 2019 2019).

Conversely, research indicates many MHapps have features that may hinder their apps' effectiveness (Bakker et al. 2016). Certain smartphone apps were found to perhaps be less effective because they may cause distress to the user due to constant reminders and notifications. For example, apps that prompt users to input information about their mood may remind a user that they suffer from depression or bring to their attention the issues they are having with their moods (Nicholas et al. 2015). The authors also indicate very few of the smartphone apps marketed as MHapps were grounded in evidence-based practice or adhered to clinical practice or disorder management.

\section{Safety Concerns}

Counselors have a duty to protect their clients, especially with the use of MHapps in the counseling relationship. Because MHapps are not currently regulated, counselors need to be cautious when supplementing counseling with these apps. Nicholas et al. (2015) found most MHapps are marketed as "health and fitness" or "medical applications" giving the impression that these apps are made available because they are effective. Moreover, some apps can dangerously mislead clients by claiming to cure their disorders, thus giving the impression they do not need to seek mental health treatment (APK Pure 2017). MHapps could jeopardize client safety if information is not provided, such as a disclaimer that instructs users to call 911 or seek assistance from a mental health professional in the event of an emergency situation (e.g., suicidal ideation or self-injurious behaviors). Martinengo et al. (2019) indicated more than 2 million MHapps were downloaded that did not display suicide hotline numbers or displayed incorrect numbers.

Disclaimers are statements that would inform consumers of the risks associated with using the MHapp and are significantly important, particularly in cases where the individuals seeking MHapps have suicidal ideations. For example, it is imperative MHapps provide instructions for users to contact 911 or go to the nearest hospital in the event they are in danger of harming themselves, and a disclaimer would ideally provide such information to the user. MHapps do not provide effective counseling treatment or interventions to address suicidal ideation (Donker et al. 2013; Grist et al. 2017; Nicholas et al. 2015).

While some MHapps include a set of disclaimers for the user, Martinez-Martin and Kreitmair (2018) found this information is often presented in dense, formal language. Moreover, most users do not take the time to read through the disclaimers and if they do, they usually do not fully understand the information presented to them (Böhme and Köpsell 2010; Martinez-Martin and Kreitmair 2018; Windle 2008). Disclaimers should be clear, concise, and in a language the user can understand (Kreitmair et al. 2017). For example, although the developers for Moodpath, a popular app for depression, disclose, they share data with both Google and Facebook "the disclosures are buried in the seventh section of the app's privacy policy" (Hess 2019).

Minimally, MHapps should include a disclaimer that explicitly states they are meant to compliment, not replace therapy, because their use could delay individuals from seeking traditional face-to-face counseling sooner (Prentice and Dobson 2014).

\section{Collection of Personal Information}

A common problem with MHapps is the management and manipulation of users' personal information that is collected. Information collected from apps can be used and shared with third parties, which is rarely disclosed to the user (Hess 2019). Minimal to no information is provided by app developers in regard to the ways personal information is used and shared with third parties. Information sharing is a frequent concern in MHapps. Minimal to no information is provided by app developers with regard to the ways personal information is used and shared with third parties. In fact, Herzog (2020) states, "Sharing data with third parties is ubiquitous across mental health apps" and found both Talkspace and BetterHelp, apps that provide users with online mental health counseling, share data with third parties.

A study conducted in 2019 found that more than $80 \%$ of 36 top-ranked apps for depression and smoking cessation collected and sent data to Google and Facebook "often without disclosing it in their privacy policies" (Herzog 2020; Hess 2019). Moodpath, a popular depression app in the Google and Apple app stores, was found to collect and share data with both Google and Facebook (Hess 2019).

Dehling et al. (2015) examined MHapps within Apple and Android platforms and found that $94.9 \%$ of the apps were 
found to be vulnerable to manipulation of personal information. Furthermore, Thurm and Kane (2010) concluded that $55 \%$ of apps studied were found to collect and send some of the user's personal information to other companies (e.g., marketing companies, consumer companies).

Ideally, MHapps would include disclaimers that would identify the risks and benefits of using the app with regard to the collection and use of users' personal information. However, despite the inclusion of a disclaimer, individuals may not be deterred from utilizing a MHapp, even when it explicitly states collected data is shared with third parties. According to a new survey presented by the Center for Data Innovation, 58\% of Americans voluntarily allow third parties to collect their sensitive personal data (i.e., location, biometrics, or medical data) in exchange for services (Castro and McLaughlin 2019).

Popular third-party apps often require full access to personal information before receiving access to the app. Bitmoji, a very popular third-party keyboard, is an example of an app that requires full access to a user's information, which includes "text that you've written before you enabled full access" (Peterson 2020). Therefore, Bitmoji will have access to anything a user types on the keyboard, such as Google searches, social media posts, credit card information, social security numbers, addresses, phone numbers, passwords, just to name a few. However, users accept the explicit terms and knowingly give the app full access to their personal information.

Additionally, apps can monitor mobile sensors in user's phones to collect information without public knowledge, such as GPS data, call logs, and phone activity, which is necessary to track behaviors such as mood, fatigue, social activity, and physical isolation (Place et al. 2017; Saeb et al. 2015). The rationale behind collecting this information is that GPS data may correlate with changes in emotional states, and it would provide realtime behavioral data to approximate information about mood (Ben-Zeev et al. 2015; Grünerbl et al. 2015; Palmius et al. 2017). Moreover, phone calls can be analyzed by apps to infer information about mood. A person's voice and speech during phone calls can be analyzed and recorded by biosensors in smartphone devices. GPS data, voice analysis, and speech analysis have been investigated as a passive method of examining individuals' mental states (Hopkins et al. 2005).

Most apps require a Wi-Fi connection, which further increases the risk of information being collected or "hacked" on smartphone apps. In fact, the United States Department of Health and Human Services state if a service provider fails to secure individual files and safeguard against theft or internet hackers, there may be adverse results for mental health providers (e.g., fines, suspensions, or loss of licensures) (New Rule Protects 2013).
Privacy and Confidentiality Another major concern is the privacy and confidentiality of the personal information after it is collected. Stiles-Shields et al. (2017) state that a majority of MHapps have been found to pose a threat to privacy and confidentiality. Privacy refers to being free from observation, whereas confidentiality refers to keeping information safe, secret, and private (Privacy, n.d.; Confidentiality, n.d.).

Privacy Users have the right to determine how their personal information is being shared, who may have access to their information, and how it is used (Cohn 2006; Kotz 2011). However, they rarely do with smartphone apps. For example, Apple and Android smartphone devices have cloud-based services that syncs information with your smartphones to make information accessibility more convenient. However, this seemingly convenient service makes breaching an individual's privacy easier for those who want to access the information illegally (Benkler 2016). Moreover, information can be unknowingly accessed remotely from one person's smartphone to another smartphone without any human interaction (Huckvale et al. 2015; Sarma 2015; Schneier 2016) or via trackers in apps that forward personal information to third parties while a user is asleep. Fowler (2019) stated, "IPhone apps I discovered tracking me by passing information to third parties - just while I was asleep - include Microsoft OneDrive, Intuit's Mint, Nike, Spotify, The Washington Post and IBM's the Weather Channel."

Although a disclaimer regarding privacy is recommended, Macdonald and Cranor (2008) estimated that it would take one person 201 hours to read most privacy policies on websites encountered within a year.

Confidentiality The Health Insurance Portability and Accountability Act (HIPAA) is used in medical and mental health practices to address some of the issues concerning client confidentiality. However, app developers, data storage, and processing companies do not meet the regulatory language or criteria to fall under the jurisdiction of HIPAA. Therefore, most MHapps are not regulated or required to comply with HIPAA standards (Terry and Gunter 2018). In addition, HIPAAs privacy and security rules only apply to healthcare providers and their business associates (U.S. Department of Health and Human Services 2016). Because MHapps are developed outside of traditional healthcare providers, they are not regulated by HIPAA (Terry 2016).

Encryption includes the coding and storing of information, which may help ensure information remains confidential and inaccessible by unauthorized individuals. However, many MHapps do not specify if and/or how personal information is encrypted within the app. MHapps imply that personal information, such as mood tracking, journal entries, and even interactions with live or artificial intelligent counselors, are stored on MHapp. Without proper encryption, the user's 
personal information may be accessible by other individuals/ companies, such as marketing companies and other third parties (e.g., individual's username and password, contact information, basic demographic information, and phone number). Bauer et al. (2017) stress the importance of a careful understanding of data encryption and informed consent to ensure users are not threatened by privacy violations. More specifically, the authors mention that an informed consent that clearly describes data encryption is important because, as previously mentioned, users are rarely able to understand the provided terms and conditions since they are written in legal language (Bauer et al. 2017; PCAST 2014). Price et al. (2014) suggest that MHapps include password protection to ensure information is not accessible to strangers that may gain access to the user's smartphone.

\section{Governing Bodies}

Currently, there are no governing bodies that specifically regulate the practice of MHapps (U.S. Department of Health and Human Services 2016). Furthermore, there are no requirements that guide smartphone app developers about consent, privacy protocols, and ethical practices of counseling (Martinez-Martin and Kreitmair 2018). This is of particular concern because numerous MHapps do not have privacy policies in place.

However, the Federal Trade Commission (FTC) may have jurisdiction with MHapps and general powers to regulate "unfair or deceptive acts or practices" (Federal trade commission act section 5 2016). In fact, the FTC set precedence when a company violated their privacy policy. Turn Inc. had been shown to be deceptive to their consumers by tracking them online and through their smartphone app even after the consumers opted-out of such tracking (FTC Approves Final Consent 2017). Turn Inc. stated that consumers could prevent targeted digital advertising by blocking the website's cookies. However, the company was still able to track tens of millions of consumers, even after blocking the website's cookies (FTC Approves Final Consent 2017). Furthermore, there were no opt-out options for the smartphone app.

Interestingly, the FTC has published data protection guidance for app developers. The FTC collaborated with the United States Food and Drug Administration (FDA) and the Department of Health and Human Services to create guidance for the Medicare Modernization Act developers (FTC releases new guidance 2016). In 2016, Lumosity was ordered to pay \$2 million for fraudulent claims, and the FTC has since issued a general warning that "it is on the lookout for companies cashing in on the popularity of mobile phone applications by developing dietary or medical apps with dubious claims of health benefits" (Walters 2016).

The FDA applies a similar risk-based approach to smartphone device software functions as other medical devices, to ensure safety and effectiveness (Device Software Functions 2019). However, the FDA is unclear whether MHapps fall into the scope of mobile medical apps. According to the FDA's mobile medical apps policy, "The FDA's mobile medical apps policy does not regulate the sale or general consumer use of smartphones or tablets" (Device Software Functions 2019). The FDA's mobile medical apps policy does not consider the Apple App Store or the Google Play Store to be medical device manufacturers and recently announced it would be relaxing its mental health app vetting process (Walters 2016). Hein (2019) stated, "Currently, the FDA has few guidelines for medical mobile applications (MMAs) aimed at treating mental health concerns and prefers to regulate such products as necessary." However, psychiatric professionals have raised concerns regarding the efficacy and safety of mental health-related apps, which may spur the development of regulations in the future (Hein 2019),

Although attention is being drawn to issues related to MHapps, there does not appear to be any entity overseeing or "policing" their safety and effectiveness. Hess (2019) indicated "the government may need to get involved in regulating this fast-moving marketplace," as they did when cigarette companies were forced to add a warning.

\section{Ethical Considerations for Counselors}

MHapps have the potential to positively impact the mental health care field and become a useful tool in counseling practices. However, without sufficient knowledge or training about the safety, efficacy, and ethical concerns, counselors could unintentionally harm clients when supplementing counseling with MHapps.

There are four major ethical principles counselors are bound to adhere to in all clinical interventions (e.g., autonomy, justice, beneficence, and non-maleficence) (ForesterMiller and Davis 2016). These ethical principles were established to ensure counselors provide clients with the best possible care while reducing harm. It makes sense that these ethical principles would apply to counselors' use of MHapps in counseling; however, to date, that relationship has not been established.

\section{Beneficence and Non-maleficence}

MHapps may improve the daily functioning of individuals who need mental health assistance while avoiding harm to the client. Both beneficence and non-maleficence of the four major ethical principles and the ACA Code of Ethics address "not causing harm to others" (American Counseling Association 2014; Forester-Miller and Davis 2016). In fact, several sections in the ACA Code of Ethics state that counselors have the responsibility to avoid and, if unavoidable, minimize harm to their clients (e.g., section A, section B, 
section $\mathrm{C}$, section $\mathrm{E}$, section $\mathrm{F}$, section $\mathrm{G}$, and section $\mathrm{I}$ ). Harming the user, for example, may include making false claims about the effectiveness of MHapps and failure to protect the user's information. Clients might use MHapps apps believing they are useful without proper investigation. Moreover, Section H of the Code of Ethics specifically addresses counselor's knowledge and competence regarding the use of distant counseling technology and social media use, as well as ethical and legal considerations that may impact both counselors and clients (American Counseling Association 2014).

\section{Responsibility}

This principle asserts that it is the responsibility of the counselors to provide the best care possible to their clients, including the use of technology, such as MHapps. Counselors have the responsibility to evaluate any tools or treatment options prior to administering any tools or treatment options to their clients.

\section{Justice}

Justice entails providing accessible treatment options and meeting client's needs. Certain MHapps are not available to users depending on their operating systems. For example, Apple smartphones have different MHapps for download compared to Android smartphones. It would be the counselor's responsibility to supplement counseling with apps that are accessible to the client.

\section{Professional Competency}

According to the American Counseling Association's (ACA) Code of Ethics, counselors are to practice within their boundaries of competence. Specifically, Section C.2.b. indicates counselors should receive appropriate education and training prior to practicing in specialty areas new to them, taking "steps to ensure the competence of their work and protect others from possible harm" (American Counseling Association 2014, p. 8). Integrating or supplementing counseling with technology has quickly become a specialty area and requires education and training.

Although guidelines have been developed for the use of technology in mental health counseling (American Counseling Association 2014; American Psychiatric Association 2013; Suggested Principles for the Online 2000; Steakley-Freeman 2017), guidelines pertaining to the ethical practice regarding MHapps have not been established. Section $\mathrm{H}$ in the ACA Code of Ethics provides guidelines for counselors with regard to Internet-related activities (e.g., distance counseling, technology, and social media), although it is unclear whether Internet-related technology encompasses smartphone apps considering the document's publication date. Regardless, Section H.1.a. in the ACA Code of Ethics directs counselors who use distance counseling, technology, and/or social media "develop knowledge and skills" regarding technical and ethical considerations (American Counseling Association 2014, p. 17). Therefore, it is imperative counselors adhere to their code of ethics and obtain training or education prior to supplementing counseling with MHapps to ensure their competency and clients' safety.

\section{Informed Consent}

Informed consent ensures transparency between the therapist and the client and gives the client the autonomy to decide if counseling will be beneficial for them. Furthermore, mental health counseling informed consents are intended to provide clear and concise outlines of the risks and benefits, alternative methods, and associated stipulations to the counseling relationship (Martinez-Martin and Kreitmair 2018; Nebeker et al. 2016; Prentice and Dobson 2014). Several organizations require counselors to provide an informed consent to their clients (e.g., American Psychological Association, American Counseling Association, the International Society for Mental Health Online, the National Board for Certified Counselors, and the American Medical Association). The ACA Code of Ethics is clear about the content to be included in an informed consent in a counseling context, such as risks and benefits of the therapeutic intervention, information on the counselor, privacy concerns, and options to leave counseling, to name a few (e.g., Section A, Section B, Section D, Section E, Section F, Section G, and Section H) (American Counseling Association 2014), information regarding the supplemental use or integration of MHapps in counseling is not addressed.

Although mental health professionals are required to be transparent with services provided, including risks of participating in counseling, they are not required to address MHapps in their informed consent. However, guidelines regarding users' protection of personal information and privacy are not in place for MHapp developers, and many MHapps do not include a disclaimer that informs users of potential safety and efficacy issues prior to users consenting to or accepting the terms and using the app. Grundy et al. (2019) found the vast majority of medical-related mobile apps routinely shared user data without the user's consent and state clinicians' informed consents should include information regarding the "potential for the loss of personal privacy" when recommending apps to consumers. At a minimum, including this information in a counselor's informed consent could help users become more knowledgeable when making decisions about the use of MHapps.

\section{MHapp Evaluation Tools}

As previously discussed, the volatile nature of the app marketplace makes it impossible to receive up-to-date evidencebased research findings regarding an app's effectiveness. 
Moreover, users are unlikely to visit websites and explore research supporting an app's effectiveness. Most users are likely to use apps based on word of mouth recommendations or through social media (Schueller et al. 2018). And, users may select apps based on users' reviews, which can be misleading because they are not typically based on effectiveness In fact, Kertz et al. (2017) indicated that of the 25 most popular apps for anxiety and worry in the Apple Store according to users' reviews, none included "content consistent with evidence-based treatments."

Ideally, apps would be subjected to stringent efficacy testing prior to their app marketplace debut. Therefore, it may be more practical for counselors to independently investigate MHapps of interest by exploring the app online, reviewing research databases for research findings, and seeking consultation from outside professionals. Licensed mental health counselors could also utilize well-known app evaluation tools, such as Psyberguide, the American Psychiatric Association's Application Evaluation tool, and the Mobile Application Rating Scale end user version (uMARS).

\section{Psyberguide}

The Psyberguide is a website created by a team of psychiatrists and psychologists who work to evaluate various MHapps that are available on both Android and Apple platforms. Psyberguide evaluates apps using three criteria: credibility, user experience, and transparency. The credibility score represents the strength of the scientific research that supports the app, as well as evaluates the therapeutic interventions that the app provides. User experience measures the design, accessibility, and overall experience the MHapp provides. This includes the MHapps ease of use and how well the app can be navigated. Transparency involves the clarity of the apps' privacy and policy; this would include data storage and collection procedures of users' information, as well as the apps' associated servers. Transparency also involves how accessible this information is to potential users. Psyberguide is a collaborative effort; professionals and users are encouraged to email Psyberguide with suggested smartphone apps. Psyberguide then evaluates apps that are suggested to them based on their criteria. Although an ambitious effort, it is unsure how often app information is updated, and how well this group can manage the volatile app market. And, according to Herzog (2020), Dr. Stephen Schueller, who spent more than 10 years running Psyberguide, stated only $3 \%$ of thousands of apps are evidence-based and that, "Finding an effective therapy app can be like finding a needle in a haystack.".

\section{American Psychiatric Association App Evaluation Model}

A collaborative app evaluation model was developed where professionals are encouraged to inform each other about their experience with MHapps (American Psychiatric Association 2017). This model utilizes a hierarchical rating system and rubric to help APA members be aware of important considerations when selecting a MHapp for use with patients. However, the site clearly states, "while we offer examples of how to use this model, we do not explicitly rate apps. Given that apps are constantly updating and changing, such information would quickly be out of date." Additionally, it does not provide information or research as to the items selected for the evaluation model.

There are five steps to the system: gather background information, risk/privacy and security, evidence, ease of use, and interoperability. The first step involves gathering background information about the app such as information about the developer, the platform the app is compatible with, and the date the app was last updated. The tool also includes how the app is classified (e.g., classified as a MHapp or a game?). The second step involves the risk/privacy and security of the app. This step involves reviewing the privacy policy, what and how data is collected from the user, can users opt-out of data collection, and who has access to the user's information. The third step considers the evidence supporting the app and its practices. Such as, are there any studies conducted on the effectiveness of the app, or what does the app claim to do versus what the app actually does, and what is the feedback from users? The fourth step investigates the apps' ease of use. This step considers whether the app is culturally relevant, what platforms it works on, if it can be used long-term, and if it requires a Wi-Fi connection to work. The last step involves interoperability. This step considers who owns the data, and if a user's data can be shared with other health fitness apps.

However, depending on the information one is seeking, this may or may not be an appropriate tool. In fact, the site states determining usability is subjective. Moreover, research to support its effectiveness was not found. Although the site is directed towards psychiatrists and other mental health professionals, the process to "evaluate" an app is lengthy and time consuming and potentially confusing, particularly for clients or patients, as it involves following several sets of questions on their website. If a mental health counselor were to assist a client with this evaluation tool, it would easily extend beyond the typical 50-min session.

\section{UMARS}

The Mobile Application Rating Scale (MARS) was developed by a group of psychiatrists and psychologists to provide mental health practitioners a tool for evaluating the quality of MHapps. The MARS is composed of 23-items based on four subscales (i.e., engagement, functionality, esthetics, and information quality), as well as a subjective quality rating. However, MARS requires training and expertise in the 
mental health field to administer it; therefore, Stoyanov et al. (2016) developed a simpler user version, uMARS. The uMARS is a 20 -item measure that includes the same four subscales as MARS, in addition to the subjective quality subscale. The uMARS provides a reliable measure of the apps' quality, determined by the users, as well as their feedback on the MHapps.

As previously stated, depending upon the information desired, these evaluation tools may or may not be helpful. The criteria used for the item selection is often unavailable, and some items may be irrelevant depending upon the information needed (e.g., ease of use, quality, users reviews).

\section{MHapp Review Websites}

These authors enacted a real-life situation a counselor may experience when attempting to verify the effectiveness or safety of an app without access to academic resources. The phrase "What is the best research-based anxiety app?" was entered into the Google search bar, and the first result was the Anxiety and Depression Association of American (ADAA) Reviewed Mental Health Apps website. This website rates an app's ease of use, effectiveness, personalization, interactive/feedback, and research evidence on a scale of 1 to 5 . The website had multiple app ratings, some of which demonstrated high numbers for "research evidence for treatment" and "research evidence for the app." For example, Anxiety Coach is the second app listed on the website, which has a research evidence rating of 5 out of 5 on the ratings key, although information regarding their review process and research evidence could not be located. These authors subsequently entered "Anxiety Coach app" into the Google search bar to view information regarding the app's research evidence; however, the search results did not provide any links to research testing. The ADAA Reviewed Mental Health Apps website also provides a link to view additional anxiety, depression and related disorder apps; however, the link forwards readers to the PsyberGuide website. These authors then searched for Anxiety Coach in the Apple Store using an iPhone XR. The app had 11 reviews with an overall 2.3 rating and was developed by the Mayo Clinic 7 years ago. The app's most recent update was in 2018.

Next, these authors entered "What's my M3" in a Google search bar, which is listed second on the ADAA Reviewed Mental Health Apps website and received a 5 out of 5 for research evidence on the rating key; however, research evidence could not be located on either the ADAA webpage or Whats my M3 webpage. Moreover, the app could not be found in the Apple and Android stores.

These seemingly credible sites gave the illusion MHapps reviews are research-based. For example, the Mental Health App Library website indicates "physicians and therapists have assessed each of these apps for quality, and curated them with Mental Health Adult Outpatient programming in mind," but the criteria used for the assessments is not provided. Yet other websites that provide libraries of MHapps, such as Rigorous App Assessment and How We Assess Health Apps, claim apps have been assessed or reviewed; however, the review process involves self-reported information from the developer with the intention of the app being added to the website's mental health app library. Again, information regarding research to support the review process could not be located.

In 2013, the National Health Service (NHS) developed a library of "safe and trusted" health apps, 14 of which were described as treating anxiety or depression (Anthes 2016). However, Huckvale et al. (2015) discovered that 35 of the MHapps cited on the NHS list of trusted apps were sharing users' identifying information (i.e., names, birthdates, e-mail addresses) to third parties, and $66 \%$ of the 35 MHapps did not encrypt their data. Therefore, in 2014, the NHS took the "safe and trusted" MHapp list offline and replaced it with a smaller list of recommended online services (Anthes 2016).

Although the purpose of websites such as those described above appear intended to credibly review MHapps, there is little to no research available regarding the app and the apps may not be available in app stores. All things considered, libraries and lists of "safe and trusted" MHapps are not reliable sources of information when considering apps for supplemental use in mental health counseling.

Trusted entities, such as the U.S. Department of Veterans Affairs (VA), also offer MHapps. Their website lists 18 MHapps, but all were developed by or in collaboration with the VA. The site highlights four top mental health apps, but research supporting the apps effectiveness or way apps were selected to be a "top mental health app" was not provided. And, although the COVID Coach app provides a brief disclaimer on the website indicating it is not intended to replace professional care, app descriptions for PTSD Coach and PTSD Family Coach do not. However, the PTSD Coach mobile app provides a software end user license agreement (EULA) that includes a privacy notice and disclaimer that must be accepted prior to using the app which states it is not intended to replace professional care and advises users to seek medical assistance in the event of an emergency. Furthermore, like most MHapps, the app was found in the health and fitness category in the Apple store.

Due to the difficulty locating information on the Internet regarding the effectiveness of MHapps, and in lieu of a timesensitive research-based tool to independently assess or evaluate MHapps prior to use, these authors developed a nonexhaustive list of items for counselors to consider prior to supplementing counseling with MHapps. This compilation of items for consideration is not intended to replace empirical research conducted to determine an app's efficacy or safety, or for the use of app endorsement or recommendations. Users are encouraged to seek consultation for clarification or questions as needed regarding any of the items. While many of the items 
overlap with those on the APA evaluation tool, several items (denoted with an asterisk in Appendix 1) were selected subsequent to the authors' research and professional experiences with technology in mental health counseling. Definitions of terms users may not be familiar with are included in Appendix 2.

\section{Discussion}

The findings from this review highlight numerous issues counselors should be aware of before supplementing counseling with MHapps. While MHapps have the potential to greatly enhance and enrich the counseling relationship, a large proportion of MHapps available to consumers have not been empirically researched to determine efficacy; however, a number of seemingly credible websites claim to have assessed or evaluated apps research evidence. This problem is compounded by the inability to provide up-to-date research findings on MHapps due to the volatile nature of the app market, or because apps may be outdated, updated, or removed from app stores. Despite the dearth of research regarding MHapp effectiveness, research has identified problems involving the use of MHapps (e.g., constant reminders and notifications, not receiving the promised results). In other words, although there is limited research to establish the effectiveness of MHapps, research has identified factors that may hinder an app's effectiveness.

Furthermore, finding a relevant app that addresses clients' specific needs can be challenging, particularly because app descriptions can be misleading, or falsely marketed altogether. Larsen et al. (2016) found that with the plethora of apps available to download for mental health purposes, finding a relevant app for consumers' specific needs can be very difficult. Shen et al. (2015) stated, "Without guidance, finding an appropriate depression app may be challenging, as the search results yielded non-depression-specific apps to depression apps at a 3:1 ratio." This problem is compounded for clients diagnosed with comorbid conditions who attempt to find a helpful MHapp. Individuals who present with two or more clinical disorders (e.g., depression and substance abuse) as identified as Diagnostic and Statistical Manual 5th edition (DSM-5) (American Psychiatric Association 2013) are considered to be experiencing comorbid conditions. Therefore, an individual may download a MHapp for depression, when in fact they might benefit more from an app that addresses substance abuse. Because MHapps do not have the ability to discern between disorders, the individual would not receive the proper treatment.

To demonstrate this point, Larsen et al. (2016) conducted a search for "depression" apps which returned 982 results. Of those, nine apps claimed to be clinically effective; however, only three of those nine apps had published findings regarding effectiveness. Therefore, only $0.003 \%$ of the 982 depression apps were found to be clinically effective. Moreover, the authors of this paper conducted a Google search for "depression app" in 2020 which returned 599,000 website; a subsequent search for "depression app" and "cure" returned 590,000 websites.

Considering the efficacy and safety concerns associated with MHapps, it is imperative licensed mental health counselors who supplement counseling with MHapps adhere to the ACA Code of Ethics to provide clients with the best possible care while diminishing harm. In the event a counselor unknowingly or inadvertently subjects their client to harm by engaging in counseling activities outside their scope of practice (e.g., endorsing an app without evidence-based research to support its effectiveness), they could violate Section A.4.a. of the ACA Code of Ethics regarding "do no harm" or Section C.2.a. (American Counseling Association 2014). Ethical violations can result in sanctions against counselors or the revocation of counseling licenses.

Without attending to the efficacy, safety, and ethical issues related to the use of MHapps, licensed mental health counselors may jeopardize their client's safety, as well as their own career should ethical violations occur. And, without a governing body to oversee the development and use of MHapps, the responsibility for ethical use and client safety rests solely on the licensed mental health counselor. Therefore, it is advisable for counselors to include information related to MHapps in their informed consent.

It is also imperative that jurisdiction is identified or created to govern the development and use of MHapps. At a minimum, MHapps should be required to be more transparent and include disclaimers that alert users to potential collection of private information, information about the developers (i.e., developer's name and credentials, mental health affiliation), and where users can obtain information about or evaluation methods or efficacy testing. Additionally, it is of vital importance that MHapps include a disclosure to protect app users, as well as developers, that states the app is not intended to replace mental health counseling and to seek professional help in the event of an emergency (e.g., go to the nearest emergency room, call 911 in the USA). After all, warning labels to avoid liability and injury or failure to warn lawsuits are required for most potentially harmful products in the USA. It seems reasonable they would be required for products that are potentially harmful, often free of charge, and easily accessible to a wide range of users that include individuals with serious mental disorders and minors.

As previously stated, the U.S. Department of Veteran's Affairs (VA) website has an app store which lists a small variety of apps, 18 of which are MHapps. Of note, though, is the VA's PTSD Coach mobile app, which provides an easy to understand End User Licensing Agreement (EULA) that could serve as a guide for other MHapps. In part, it states the following: 
The content of this application is intended for use only as an informative tool. It is not, is not intended to be, and should not be used in any way as a substitute for professional medical advice or training. The accuracy of the information provided is not guaranteed. You acknowledge in initiating this application that the information is not meant to diagnose a health condition or disease and is not meant to develop a health treatment plan. If you are in an emergency or life-threatening medical situation, seek medical assistance immediately. Dial emergency number (911 in the USA) for emergency medical services.

Disclosures such as this would address some of the safety concerns highlighted in this article that need immediate attention to protect users from potential harm.

\section{Implications}

A substantial concern for mental health counselors regarding client safety is the absence of informed consents in MHapps. In order to provide transparent services, it would behoove mental health counselors to include a statement that at a minimum addresses the potential loss of privacy or unintended data collection associated with the supplemental use of MHapps. Counselors will want to provide clients with an opportunity to ask questions regarding this matter to help them understand some of the risks they may be exposed to.

Additionally, without a central location or database to obtain up-to-date research-based information about MHapps, it is unlikely mental health counselors will be able to access research about an app's effectiveness, particularly if they do not have access to academic databases. Simply completing an Internet search for information about a MHapp's effectiveness or research will likely result in various misleading and vague outcomes, which hinders counselors' ability to make informed decisions about supplementing counseling with MHapps. Many seemingly credible websites claim to have reviewed or assessed apps, but information about the method or way(s) apps were reviewed or assessed is not provided on the website. Therefore, these websites give the illusion apps are safe and effective because they have been reviewed or assessed, but it is unclear if and how their effectiveness was tested.

It is clear further research is warranted regarding the efficacy of MHapps, client safety, and potential liability pitfalls licensed mental health counselors may encounter when supplementing counseling with MHapps. In lieu of a timesensitive research-based tool to independently assess or evaluate MHapps prior to use, these authors developed a nonexhaustive list of items to consider prior to using or supplementing counseling with MHapps (see Appendix 1). It is important to note that this compilation of items for consideration is not intended to replace empirical research to determine an app's efficacy or safety, or for the use of app endorsement or recommendations. Users are encouraged to seek professional consultation for clarification or questions as needed regarding any of the items.

\section{Conclusion}

MHapps have the potential to radically improve the lives of many people, especially those receiving mental health services. The use of MHapps have the capacity to become a useful tool in counseling practices, as well as assist clients with managing their symptoms. However, these apps pose many challenges to licensed mental health counselors. MHapps lack ethical guidelines, pose threats to confidentiality and privacy, and lack scientific evidence. Due to these limitations, it would behoove counselors to become knowledgeable about the safety and ethical issues surrounding the supplemental use of MHapps in counseling. Counselors can then help clients make informed decisions when choosing an MHapp. Despite the minimal research that has been conducted regarding the efficacy of MHapp, various tools and models are available to assist users when evaluating the effectiveness of MHapps (e.g., Psyberguide, American Psychiatric Association App Evaluation Model, and uMARS). Furthermore, the authors of this article compiled a list of items for licensed mental health counselors to consider prior to supplementing counseling with MHapps.

Acknowledgments The corresponding author is thankful to Varsha Varghese, HBSc, for her assistance with data collection.

\section{Compliance with Ethical Standards}

Conflict of Interest The authors declare that there is no conflict of interest.

\section{Appendix 1. Items for licensed mental health counselors to consider prior to using a MHapp}

Disclaimer: In lieu of a time-sensitive research-based tool to independently assess or evaluate MHapps prior to use, following is a non-exhaustive list of items to consider prior to using or supplementing counseling with MHapps. This compilation of items for consideration is not intended to replace empirical research to determine an app's efficacy or safety, or for the use of app endorsement or recommendations. Seek consultation for clarification or questions as needed regarding any of the items.

1. Is the app compatible with the smartphone platform (i.e. Apple or Android)?* 
2. What is the classification of the app (e.g. health, gaming)?*

3. Is the app free? Or are there fees to access additional app options? *

4. Does it require WIFI to work? *

5. Is there any feedback from users to support claims (e.g. app store reviews, websites)? *

6. Does the application have impossible claims ("cures depression")? *

7. What is the credibility of the developers or are they mental health affiliated? *

8. Have studies been conducted demonstrating the app's efficacy? If so, has the app been updated since that time? *

9. Are there other apps created by the developer? If so, what are they?

10. Is personal information required to use the app? If so, is personal data encrypted or de-identified? *

11. Is there a privacy policy or disclaimer that addresses how personal information is collected and used? *

12. Does it claim to meet HIPAA standards? *

13. Does it include an informed consent?

14. Is data shared with outside third parties? *

15. Can the app share data with other user tools (Apple Healthkit, Fitbit)? *

16. Can you opt out of and/or delete data collection? *

17. Does the app use cookies (e.g. tracking of personal information)? *

18. Is it customizable? *

19. Is the application easy to access for the patient and/or accessible for those with impaired vision or other disabilities? *

20. Can counselors or clients export/download personal data? *

21. Are there emergency instructions available (e.g. 911, suicide hotline)?

22. Is the app intended to be used alone or in conjunction with therapy?

*Denotes items that overlap with the APA evaluation tool.

\section{Appendix 2. Definitions of terms}

Consent: to provide approval or agreement (Consent, n.d.).

Cookies: a file that is stored on a World Wide Web user's computer, which is both created and used by websites servers and contains personal information (i.e. preferences, browsing history) (Cookies, n.d.).

Efficacy: the ability to produce an effect (Efficacy, n.d.).

Encrypted: to encipher; to convert a message into code (Encrypt, n.d.; Encipher, n.d.).

End User License Agreement (EULA): a legal contract between a software developer and a user, where the user can either accept or reject (Rouse 2005).
HIPAA: an acronym for the Health Insurance Portability and Accountability Act, which is a US law created to provide privacy standards to protect patient's information and medical records in the health professions (Shiel 2018).

Informed Consent: an individual's consent to participate in a health care intervention, in which the individual has a full understanding of what is involved and the possible consequences (Informed Consent, n.d.).

Privacy: freedom from unsanctioned invasion and intervention (Privacy, n.d.).

Third parties: an individual or entity involved in a transaction other than the principals (Third Parties, n.d.)

\section{References}

Ahmad, I. (2019). 60+ fascinating smartphone apps usage statistics for 2019 [infographic] Retrieved from https://www.socialmediatoday. $\mathrm{com} /$ news/60-fascinating-smartphone-apps-usage-statistics-for2019-infographic/550990/

American Counseling Association. (2014). ACA code of ethics. Alexandria, VA:

American Psychiatric Association. (2013). Diagnostic and statistical manual of mental disorders (5th ed.). Retrieved from https://doi. org/10.1176/appi.books.9780890425596

American Psychiatric Association. (2017). App evaluation model. Retrieved from https://www.psychiatry.org/psychiatrists/practice/ mental-health-apps/app-evaluation-model

Anthes, E. (2016). Mental health: there's an app for that. Nature, 532(7597), 20. Retrieved from https://www.ncbi.nlm.nih.gov/ pubmed/27078548.

APK pure. (2017). Retrieved from https://apkpure.ai/cure-anxiety-anddepression

Bakker, D., Kazantzis, N., Rickwood, D., \& Rickard, N. (2016). Mental health smartphone apps: review and evidence-based recommendations for future developments. JMIR Mental Health, 3(1) Retrieved from https://doi.org/10.2196/mental.4984, e7

Bakker, D., \& Rickard, N. (2018). Engagement in mobile phone app for self-monitoring of emotional wellbeing predicts changes in mental health: MoodPrism. Journal of Affective Disorders, 227, 432-442. https://doi.org/10.1016/j.jad.2017.11.016.

Bauer, M., Glenn, T., Monteith, S., Bauer, R., Whybrow, P., \& Geddes, J. (2017). Ethical perspectives on recommending digital technology for patients with mental illness. International Journal of Bipolar Disorders, 5(1), 1-14. https://doi.org/10.1186/s40345-017-0073-9.

Benkler, Y. (2016). Degrees of freedom, dimensions of power. Daedalus, 145(1), 18-32. https://doi.org/10.1162/DAED_a_00362.

Ben-Zeev, D., Scherer, E. A., Wang, R., Xie, H., \& Campbell, A. T. (2015). Next-generation psychiatric assessment: using smartphone sensors to monitor behavior and mental health. Psychiatric Rehabilitation Journal, 38(3), 218-226. https://doi.org/10.1037/ prj0000130.

Böhme, R., \& Köpsell, S. (2010). Trained to accept? Paper presented at the 2403-2406. doi:https://doi.org/10.1145/1753326.1753689 Retrieved from http://dl.acm.org/citation.cfm?id=1753689

Castro, D., \& McLaughlin, M. (2019). Survey: majority of Americans willing to share their most sensitive personal data. Retrieved from https://www.datainnovation.org/2019/01/survey-majority-ofamericans-willing-to-share-their-most-sensitive-personal-data/

Clay, R. (2020). Using apps with your patients. Retrieved from https:// www.apa.org/monitor/2020/04/career-using-apps 
Clement, J. (2019a). Annual number of global mobile app downloads 2016-2018. Retrieved from https:/www.statista.com/statistics/ 271644/worldwide-free-and-paid-mobile-app-store-downloads/

Clement, J. (2019b). Apple: most popular app store categories 2019. Retrieved from https:/www.statista.com/statistics/270291/popularcategories-in-the-app-store/

Clement, J. (2019c). Average frequency of using an app per day according to mobile device users in the united states as of April 2018, by age group. Retrieved from https://www.statista.com/statistics/ 243856/daily-app-use-by-us-mobile-app-users/

Clement, J. (2019d). Average number of new android app releases per day from 3rd quarter 2016 to 1st quarter 2018. Retrieved from https:/www.statista.com/statistics/276703/android-app-releasesworldwide/

Clement, J. (2019e). Average number of new iOS app releases per day from 3rd quarter 2016 to 1 st quarter 2018. Retrieved from https:// www.statista.com/statistics/276705/ios-app-releases-worldwide/

Clement, J. (2019f). Number of apps available in leading app stores as of 3rd quarter 2019. Retrieved from https://www.statista.com/statistics/ 276623/number-of-apps-available-in-leading-app-stores/

Cohn, S. P. (2006). Privacy and confidentiality in the nationwide health information network. Retrieved from https://ncvhs.hhs.gov/rrp/ june-22-2006-letter-to-the-secretary-recommendations-regardingprivacy-and-confidentiality-in-the-nationwide-health-informationnetwork/

Dehling, T., Gao, F., Schneider, S., \& Sunyaev, A. (2015). Exploring the far side of mobile health: information security and privacy of mobile health apps on iOS and android. JMIR mHealth and uHealth, 3(1), e8. https://doi.org/10.2196/mhealth.3672.

Device software functions including mobile medical applications. (2019). Retrieved from https://www.fda.gov/medical-devices/digital-health/ device-software-functions-including-mobile-medical-applications\#

Donker, T., Petrie, K., Proudfoot, J., Clarke, J., Birch, M., \& Christensen, H. (2013). Smartphones for smarter delivery of mental health programs: a systematic review. Journal of Medical Internet Research, 15(11), e247. https://doi.org/10.2196/jmir.2791.

Edwards-Stewart, A., Alexander, C., Armstrong, C. M., Hoyt, T., \& O'Donohue, W. (2019). Mobile applications for client use: ethical and legal considerations. Psychological Services, 16(2), 281-285. https://doi.org/10.1037/ser0000321.

Federal trade commission act section 5: unfair or deceptive acts or practices. (2016). Retrieved from https://www.federalreserve.gov/ boarddocs/supmanual/cch/ftca.pdf

Forester-Miller, H., \& Davis, T. (2016). Practitioner's guide to ethical decision making. Retrieved from https://www.counseling.org/docs/ default-source/ethics/practioner-39-s-guide-to-ethical-decisionmaking.pdf?sfvrsn $=10$

Fowler, G.A. (2019). It's the middle of the night. Do you know who your iPhone is talking to? Retrieved from https://www.washingtonpost. com/technology/2019/05/28/its-middle-night-do-you-know-whoyour-iphone-is-talking/

FTC approves final consent order with online company charged with deceptively tracking consumers online and through mobile devices. (2017). Retrieved from https://www.ftc.gov/news-events/pressreleases/2017/04/ftc-approves-final-consent-order-online-companycharged

FTC releases new guidance for developers of mobile health apps. (2016). Retrieved from https://www.ftc.gov/news-events/press-releases/ 2016/04/ftc-releases-new-guidance-developers-mobile-health-apps

Grist, R., Porter, J., \& Stallard, P. (2017). Mental health mobile apps for preadolescents and adolescents: a systematic review. Journal of Medical Internet Research, 19(5), e176. https://doi.org/10.2196/ jmir.7332

Grundy, Q., Chiu, K., Held, F., Continella, A., Bero, L., \& Holz, R. (2019). Data sharing practices of medicines related apps and the mobile ecosystem: traffic, content, and network analysis. $B m j$, 364, 1920. https://doi.org/10.1136/bmj.1920.

Grünerbl, A., Muaremi, A., Osmani, V., Bahle, G., Ohler, S., Troster, G., et al. (2015). Smartphone-based recognition of states and state changes in bipolar disorder patients. IEEE Journal of Biomedical and Health Informatics, 19(1), 140-148. https://doi.org/10.1109/ JBHI.2014.2343154.

Hein, A. (2019). How does the FDA regulate mental health apps? Retrieved from https://clarkstonconsulting.com/insights/mentalhealth-apps-fda/

Herzog, K. (2020). Mental health apps draw wave of new users as experts call for more oversight. Retrieved from https://www.cnbc.com/ 2020/05/24/mental-health-apps-draw-wave-of-users-as-expertscall-for-oversight.html

Hess, P. (2019). Privacy concerns about mental health apps highlight need for regulation. Retrieved from https://www.spectrumnews. org/news/mental-health-apps-highlight-need-for-regulation/

Hopkins, C. S., Ratley, R. J., Benincasa, D. S., \& Grieco, J. J. (2005). Evaluation of voice stress analysis technology. Paper presented at the 20b. doi:https://doi.org/10.1109/HICSS.2005.254 Retrieved from https://ieeexplore.ieee.org/document/1385268

Huckvale, K., Prieto, J. T., Tilney, M., Benghozi, P., \& Car, J. (2015). Unaddressed privacy risks in accredited health and wellness apps: a cross-sectional systematic assessment. BMC Medicine, 13(1), 214. https://doi.org/10.1186/s12916-015-0444-y.

Jones, N., \& Moffitt, M. (2016). Ethical guidelines for mobile app development within health and mental health fields. Professional Psychology: Research and Practice, 47(2), 155-162. https://doi. org/10.1037/pro0000069.

Kertz, S., MacLaren Kelly, J., Stevens, K., Schrock, M., \& Danitz, S. (2017). A review of free iPhone applications designed to target anxiety and worry. Journal of Technology in Behavioral Science, 2(2), 61-70. https://doi.org/10.1007/s41347-016-0006-y.

Khalaf, S., \& Lali Kesiraju. (2017). U.S. consumers time-spent on mobile crosses 5 hours a day. Retrieved from https://www.flurry.com/post/ 157921590345/us-consumers-time-spent-on-mobile-crosses-5

Kotz, D. (2011). A threat taxonomy for mHealth privacy. Paper presented at the 1-6. doi:https://doi.org/10.1109/COMSNETS.2011.5716518 Retrieved from https://ieeexplore.iee.org/document/5716518

Krebs, P., \& Duncan, D. T. (2015). Health app use among US mobile phone owners: a national survey. JMIR mHealth and uHealth, 3(4), e101. https://doi.org/10.2196/mhealth.4924.

Kreitmair, K. V., Cho, M. K., \& Magnus, D. C. (2017). Consent and engagement, security, and authentic living using wearable and mobile health technology. Nature Biotechnology, 35(7), 617-620. https://doi.org/10.1038/nbt.3887.

Larsen, M. E., Nicholas, J., \& Christensen, H. (2016). Quantifying app store dynamics: longitudinal tracking of mental health apps. JMIR mHealth and uHealth, 4(3), e96. https://doi.org/10.2196/mhealth. 6020.

Leigh, S., \& Flatt, S. (2015). App-based psychological interventions: friend or foe? Evidence Based Mental Health, 18(4), 97-99. https://doi.org/10.1136/eb-2015-102203.

Lindhiem, O., Bennett, C. B., Rosen, D., \& Silk, J. (2015). Mobile technology boosts the effectiveness of psychotherapy and behavioral interventions. Behavior Modification, 39(6), 785-804. https://doi. org/10.1177/0145445515595198.

Liquid State. (2018). The rise of mHealth apps: a market snapshot. Retrieved from https://liquid-state.com/mhealth-apps-marketsnapshot/

Lui, J. H. L., Marcus, D. K., \& Barry, C. T. (2017). Evidence-based apps? A review of mental health mobile applications in a psychotherapy context. Professional Psychology: Research and Practice, 48(3), 199-210. https://doi.org/10.1037/pro0000122. 
Macdonald, A. M., \& Cranor, L. F. (2008). The cost of reading privacy policies. I/S: A Journal of Law and Policy for the Information Society, 4(3), 1-21.

Marshall, J. M., Dunstan, D. A., \& Bartik, W. (2019a). Smartphone psychology: new approaches towards safe and efficacious mobile mental health apps. Professional Psychology: Research and Practice, doi:https://doi.org/10.1037/pro0000278.

Marshall, J. M., Dunstan, D. A., \& Bartik, W. (2019b). Smartphone psychology: new approaches towards safe and efficacious mobile mental health apps. Professional Psychology: Research and Practice, doi:https://doi.org/10.1037/pro0000278

Marshall, J. M., Dunstan, D. A., \& Bartik, W. (2020). Clinical or gimmickal: the use and effectiveness of mobile mental health apps for treating anxiety and depression. London, England: SAGE Publications. doi:https://doi.org/10.1177/0004867419876700

Martinengo, L., Van Galen, L., Lum, E., Kowalski, M., Subramaniam, M., \& Car, J. (2019). Suicide prevention and depression apps' suicide risk assessment and management: a systematic assessment of adherence to clinical guidelines. BMC Medicine, 17(1), 231. https:// doi.org/10.1186/s12916-019-1461-z.

Martinez-Martin, N., \& Kreitmair, K. (2018). Ethical issues for direct-toconsumer digital psychotherapy apps: addressing accountability, data protection, and consent. JMIR Mental Health, 5(2), e32. https:// doi.org/10.2196/mental.9423.

Nebeker, C., Lagare, T., Takemoto, M., Lewars, B., Crist, K., Bloss, C., \& Kerr, J. (2016). Engaging research participants to inform the ethical conduct of mobile imaging, pervasive sensing, and location tracking research. Translational Behavioral Medicine, 6(4), 577586. https://doi.org/10.1007/s13142-016-0426-4.

New rule protects patient privacy, secures health information. (2013). Retrieved from https://wayback.archive-it.org/3926/ 20170118235404/https://www.hhs.gov/about/news/2013/01/17/ new-rule-protects-patient-privacy-secures-health-information.html

Nicholas, J., Larsen, M. E., Proudfoot, J., \& Christensen, H. (2015). Mobile apps for bipolar disorder: a systematic review of features and content quality. Journal of Medical Internet Research, 17(8), e198. https://doi.org/10.2196/jmir.4581.

Palmius, N., Tsanas, A., Saunders, K. E. A., Bilderbeck, A. C., Geddes, J. R., Goodwin, G. M., \& De Vos, M. (2017). Detecting bipolar depression from geographic location data. IEEE Transactions on Biomedical Engineering, 64(8), 1761-1771. https://doi.org/10. 1109/TBME.2016.2611862.

Pappas, S. (2020). Providing care in innovative ways. Retrieved from https://www.apa.org/monitor/2020/01/cover-trends-innovativeways

PCAST (President's Council of Advisors on Science and Technology). (2014). Big data and privacy: a technological perspective Retrieved from https://bigdatawg.nist.gov/pdf/pcast_big_data_and_privacy_may 2014.pdf

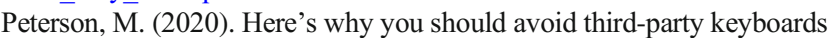
on your iPhone or iPad. Retrieved from https://appletoolbox.com/ heres-why-you-should-avoid-third-party-keyboards-on-youriphone-or-ipad/

Pew Research Center. (2019). Mobile fact sheet. Retrieved from https:// www.pewinternet.org/fact-sheet/mobile/

Place, S., Blanch-Hartigan, D., Rubin, C., Gorrostieta, C., Mead, C., Kane, J., Marx, B. P., Feast, J., Deckersbach, T., Pentland, A. “. S.”., Nierenberg, A., \& Azarbayejani, A. (2017). Behavioral indicators on a mobile sensing platform predict clinically validated psychiatric symptoms of mood and anxiety disorders. Journal of Medical Internet Research, 19(3), e75. https://doi.org/10.2196/ jmir.6678.

Prentice, J. L., \& Dobson, K. S. (2014). A review of the risks and benefits associated with mobile phone applications for psychological interventions. Canadian Psychology/Psychologie Canadienne, 55(4), 282-290. https://doi.org/10.1037/a0038113.
Price, M., Yuen, E. K., Goetter, E. M., Herbert, J. D., Forman, E. M., Acierno, R., \& Ruggiero, K. J. (2014). mHealth: a mechanism to deliver more accessible, more effective mental health care. Clinical Psychology \& Psychotherapy, 21(5), 427-436. https://doi.org/10. 1002/cpp.1855.

Rathbone, A. L., \& Prescott, J. (2017). The use of mobile apps and SMS messaging as physical and mental health interventions: systematic review. Journal of Medical Internet Research, 19(8), e295. https:// doi.org/10.2196/jmir.7740.

Reamer, F. G. (2015). Clinical social work in a digital environment: ethical and risk-management challenges. Clinical Social Work Journal, 43(2), 120-132. https://doi.org/10.1007/s10615-0140495-0.

Rouse, M. (2005). End user license agreement (EULA). Retrieved from https://searchcio.techtarget.com/definition/End-User-LicenseAgreement

Rouse, M. (2018). mHealth (mobile health) Retrieved from https:// searchhealthit.techtarget.com/definition/mHealth

Saeb, S., Zhang, M., Karr, C. J., Schueller, S. M., Corden, M. E., Kording, K. P., \& Mohr, D. C. (2015). Mobile phone sensor correlates of depressive symptom severity in daily-life behavior: an exploratory study. Journal of Medical Internet Research, 17(7), e175. https://doi.org/10.2196/jmir.4273.

Sarma, S. (2015). I helped invent the internet of things. here's why I'm worried about how secure it is. Retrieved from https://www.politico. com/agenda/story/2015/06/internet-of-things-privacy-riskssecurity-000096

Schneier, B. (2016). The internet of things will turn large scale hacks into real work disasters. Retrieved from https://motherboard.vice.com/ en_us/article/qkjzwp/the-internet-of-things-will-cause-the-firstever-large-scale-internet-disaster

Schueller, S. M., Neary, M., O'Loughlin, K., \& Adkins, E. C. (2018). Discovery of and interest in health apps among those with mental health needs: survey and focus group study. Journal of Medical Internet Research, 20(6), e10141. https://doi.org/10.2196/10141.

Shen, N., Levitan, M., Johnson, A., Bender, J. L., Hamilton-Page, M., Jadad, A. A. R., \& Wiljer, D. (2015). Finding a depression app: a review and content analysis of the depression app marketplace. JMIR mHealth and uHealth, 3(1), e16. https://doi.org/10.2196/ mhealth.3713.

Shiel, W. (2018). Medical definition of HIPAA. Retrieved from https:// www.medicinenet.com/script/main/art.asp?articlekey=31785

The state of mobile in 2019 (2019). Retrieved from https://s3.amazonaws. com/files.appannie.com/reports/1901_State_of_Mobile_Main_EN. pdf

Steakley-Freeman, D. (2017). The mental health app guide designed with you in mind. Retrieved from https://psyberguide.org/apps/

Stiles-Shields, C., Montague, E., Lattie, E. G., Kwasny, M. J., \& Mohr, D. C. (2017). What might get in the way: barriers to the use of apps for depression. Digital Health, 3, 205520761771382. https://doi. org/10.1177/2055207617713827.

Stoyanov, S. R., Hides, L., Kavanagh, D. J., \& Wilson, H. (2016). Development and validation of the user version of the mobile application rating scale (uMARS). JMIR mHealth and uHealth, 4(2), e72. https://doi.org/10.2196/mhealth.5849.

Stratton, E., Lampit, A., Choi, I., Calvo, R. A., Harvey, S. B., \& Glozier, N. (2017). Effectiveness of eHealth interventions for reducing mental health conditions in employees: a systematic review and metaanalysis. PLoS One, 12(12), e0189904. https://doi.org/10.1371/ journal.pone.0189904.

Suggested principles for the online provision of mental health services. (2000). Retrieved from https://ismho.org/resources/archive/ suggested-principles-for-the-online-provision-of-mental-healthservices/\#Consent 
Terry, N. P. (2016). Will the internet of things transform healthcare? Vanderbilt Journal of Entertainment and Technology Law, 19(2), 327.

Terry, N. P., \& Gunter, T. D. (2018). Regulating mobile mental health apps. Behavioral Sciences \& the Law, 36(2), 136-144. https://doi. org/10.1002/bsl.2339.

Thurm, S., \& Kane, Y. I. (2010). Your apps are watching you Retrieved f r o m h t p s : / / w w w. w s j.c o m / a r i c le s / SB10001424052748704694004576020083703574602

Torous, J., \& Roberts, L. (2017). The ethical use of mobile health technology in clinical psychiatry. The Journal of Nervous and Mental Disease, 205(1), 4-8. https://doi.org/10.1097/NMD. 0000000000000596.

U.S. Department of Health and Human Services, National Institutes of Health (2016). Examining oversight of the privacy \& security of health data collected by entities not regulated by HIPAA. Retrieved from https://www.healthit.gov/sites/default/files/noncovered_entities_report_june_17_2016.pdf

Vempaty, A. (2019). 20 crazy effective mobile retention tactics. Retrieved from https://amplitude.com/blog/2016/02/18/mobileretention-tactics
Walters, J. (2016). Lumosity fined millions for making false claims about brain health benefits. Retrieved from https://www.theguardian.com/ technology/2016/jan/06/lumosity-fined-false-claims-brain-trainingonline-games-mental-health

Wang, K., Varma, D. S., \& Prosperi, M. (2018). A systematic review of the effectiveness of mobile apps for monitoring and management of mental health symptoms or disorders. Journal of Psychiatric Research, 107, 73-78. https://doi.org/10.1016/j.jpsychires.2018. 10.006 .

Watson, I. (2018). Unmasking uninstalls: three data points to think about. Retrieved from https://www.adjust.com/blog/unmasking-uninstalls/

Watts, S., Mackenzie, A., Thomas, C., Griskaitis, A., Mewton, L., Williams, A., \& Andrews, G. (2013). CBT for depression: a pilot RCT comparing mobile phone vs. computer. BMC Psychiatry, 13(1), 49. doi:https://doi.org/10.1186/1471-244X-13-49.

Windle, P. E. (2008). Understanding informed consent: significant and valuable information. Journal of Perianesthesia Nursing, 23(6), 430-433. https://doi.org/10.1016/j.jopan.2008.09.004.

Publisher's Note Springer Nature remains neutral with regard to jurisdictional claims in published maps and institutional affiliations. 